\title{
Splenic Artery Embolization for Massive Splenomegaly
}

\author{
Di Martino Marcello*, Mora-Guzmán Ismael, Mingo Basail Alberto and Sánchez-Urdazpal Luis \\ Hospital Universitario de La Princesa, Madrid, Spain
}

Received: November 30, 2016; Accepted: December 15, 2016; Published: January 27, 2017

*Corresponding author: Marcello Di Martino, Hospital Universitario de La Princesa, Calle Diego de Leon, 62, 28006, Madrid, Spain; Tel: +34654583554; E-mail: marcellodima@gmail.com

\begin{abstract}
Massive splenomegaly is related to several challenges for the surgeon and higher intraoperative complications, due to the limited working space, the difficulties in spleen manoeuvre and the increase in bleeding. A splenic artery embolization can be performed before the surgery, in order to decrease both the size of the spleen and the risk of bleeding.

Here, we report the case of a 56-year-old man diagnosed with massive splenomegaly, secondary to a marginal splenic lymphoma, which had a maximum diameter of $54 \mathrm{~cm}$. The surgery was recommended because of progressive anemia requiring monthly transfusion of red blood cells, thrombocytopenia, abdominal pain and constitutional symptoms. A splenic artery embolization was undertaken just prior to the splenectomy, in order to achieve a decrease in spleen size and to reduce the risk of intraoperative bleeding. The surgery was performed uneventfully. The diameters of the resected spleen were $48 \times 24 \times 11 \mathrm{~cm}$ and its weight was 6,300 g. The splenic artery embolization is a reliable preoperative procedure before surgery for massive splenomegaly.
\end{abstract}

Keywords: Splenomegaly; Splenectomy; Massive splenomegaly; Splenic artery embolization

\section{Abbreviations}

MS: massive splenomegaly; SAE: splenic artery embolization; CT: computed tomography

\section{Introduction}

Massive splenomegaly (MS) has been defined as a spleen $>17$ $20 \mathrm{~cm}$ in length or $>1000-1,500 \mathrm{~g}$ in weight $[1,2]$. Splenectomy for MS presents several challenges for the surgeon; therefore, in order to reduce intraoperative complications, decreasing both the size of the spleen and the risk of bleeding, a splenic artery embolization (SAE) can be performed before the surgery.

Here, we report a case of MS with a weight of 6,300 g and a diameter of $48 \times 24 \times 11 \mathrm{~cm}$ submitted to splenectomy combined with SAE.

\section{Case report}

A 56-year-old man with marginal splenic lymphoma was recommended for splenectomy because of progressive anaemia
(4.5 g/dL), requiring monthly transfusion of red blood cells, leucocytosis $(107,000 / \mathrm{mm} 3)$, thrombocytopenia $(63,000 /$ $\mathrm{mm}^{3}$ ), abdominal pain and constitutional symptoms. Clinical exam showed a massive splenomegaly that overtook the midline and reached the hypogastric region. Preoperative computed tomography (CT), performed 6 months before the surgery, confirmed the enlarged spleen with a diameter of $54 \times 28 \times 14$ $\mathrm{cm}$ associated with moderate ascites, bilateral pleural effusion and enlarged retroperitoneal lymph nodes (Fig. 1). Bone marrow aspiration demonstrated a low-grade B lymphoproliferative disorder, CD5 +, CD19+ and CD20+. A complete splenic artery embolization (SAE) was performed three hours prior to surgery. The catheter tip, inserted through the femoral artery, was advanced to the splenic artery distal to the gastroepiploic artery, and Polyvinil Alcohol Foam of $700-100$ microns, coils IMWCE-35-15-10 COOK and an Amplazter Vascular Plug 4 were injected inside the vessel (Fig 2A). A posterior angiogram was performed to verify the complete occlusion of the splenic artery (Fig 2B). The patient did not complain of pain, and an evident decrease in spleen size was observed on physical examination

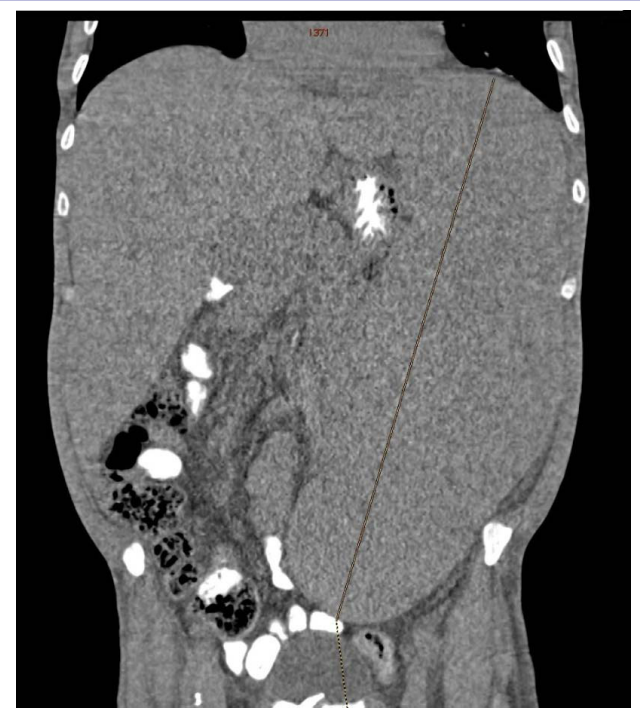

Figure 1: Computed tomography showing a coronal section of the giant abdominal mass reaching the pelvis. 
(Fig. 3). The patient was placed in the left semilateral position and an open splenectomy through a left Makuuchi incision was performed without intraoperative complications. Intraoperative blood loss was $300 \mathrm{cc}$, the operative time was 180 minutes. The resected spleen weighed $6,300 \mathrm{~g}$ and had a diameter of $48 \times 24 \times 11 \mathrm{~cm}$. The pathologic report described a low-grade B lymphoproliferative disorder, CD5+, CD20+ and Bcl-2+, compatible with marginal splenic lymphoma stage IVB Ann Arbor. The post-operative course was characterized by a postoperative ileus. The total length of hospital stay was 16 days.

\section{Discussion}

Laparoscopic splenectomy, since its first report in 1991 [3], has become the gold standard for normal-sized spleen,

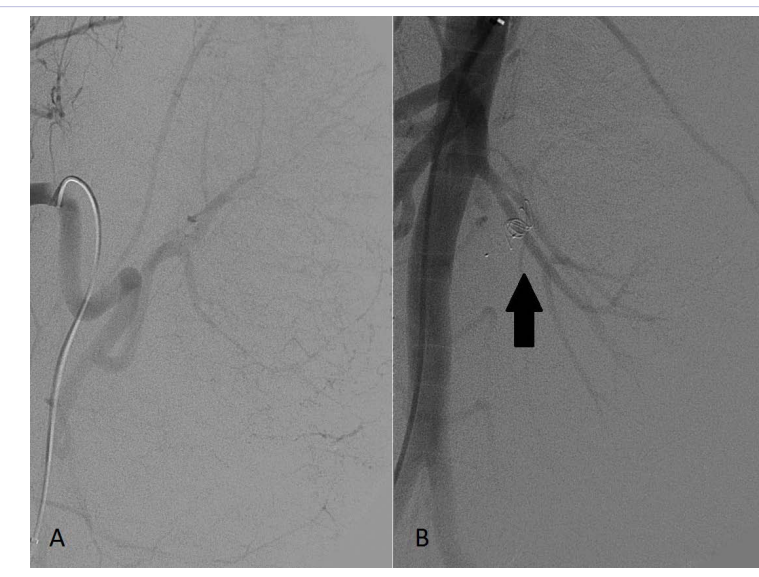

Figure 2:Angiogram realized before selective splenic artery embolization $(2 \mathrm{~A})$ and later $(2 \mathrm{~b})$, showing the coils and the Amplazter Vascular Plug in the splenic artery (black arrow)

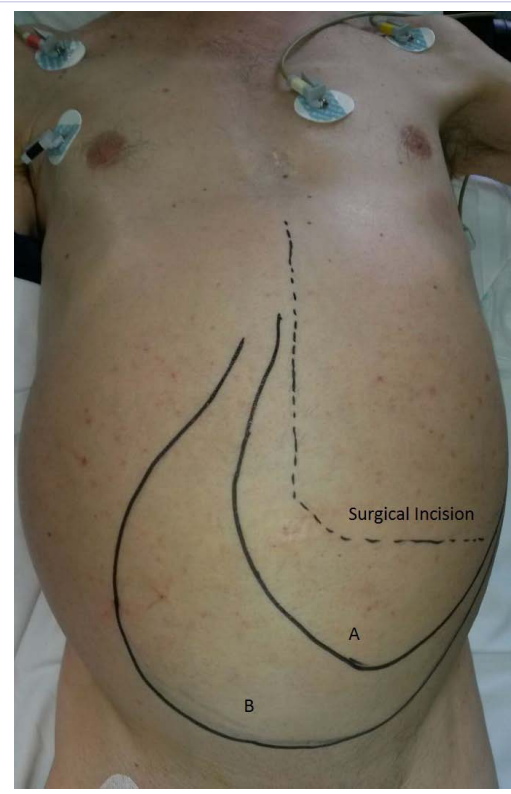

Figure 3: Spleen size before (A) and after embolization (B). although massive splenomegaly removal is associated with higher intraoperative complications compared to normalsize splenectomy. The main challenges for the surgeon are represented by the limited working space, the difficulties in spleen manoeuvre and the increase in bleeding risk, because of its peculiar vascularity. Targarona et al. [4], in their series of 186 patients submitted to splenectomy, reported that all spleen over $3.2 \mathrm{~kg}$ required conversion, although later reports have shown low conversion rate even in spleen $>3 \mathrm{~kg}$ [5]. For this reason, an open approach can be reasonably preferred in selected patients like ours, which represents one of the biggest spleen resected after SAE.

The SAE prior to surgery, firstly described by Maddison in 1973 [6], represents a reliable procedure as it can decrease spleen size, intraoperative bleeding and operative time. As shown by Iwase et al. [7], a decrease of $60 \%$ in spleen volume can be observed after SAE. In our case, a significant reduction in the size of the spleen was observed after the embolization, and this achievement considerably simplified the surgery. Naoum et al. [8] reported a significant reduction in intraoperative blood from $240 \mathrm{~mL}$ to $25 \mathrm{~mL}(\mathrm{p}<0.003)$ when SAE was performed. Even though it is generally recommended realizing a SAE for spleen > $20 \mathrm{~cm}$, a consensus about the indication of the procedure and the timing does not exist.

Complications related to SAE have been described. Postinfarction syndrome, as well as pancreatitis or pancreatic necrosis, and acute gastritis ulcers may occur [9]. Post infarction syndrome is characterized by fever and left flank pain. It has been recommended to perform the SAE the day before or on the same day as the surgery, but probably the best strategy to decrease the likelihood of uncontrolled pain is to carry out the procedure on the day of the splenectomy or combine both procedures in an intraoperative approach.

\section{Conclusion}

SAE represents a safe and feasible technique in cases of massive splenomegaly, in order to reduce the spleen size and the risk of bleeding. The laparoscopic approach should be recommended, however, in selected cases, an open approach represents a valid alternative to the extent of reducing the chances of intraoperative complications.

\section{Refrences}

1. Grahn SW, Alvarez J 3rd, Kirkwood K. Trends in laparoscopic splenectomy for massive splenomegaly. Arch Surg. 2006;141(8):755761.

2. Poulin EC, Mamazza J, Schlachta CM. Splenic artery embolization before laparoscopic splenectomy An update. Surg Endosc. 1998;12(6):870875.

3. Delaitre B, Maignien B. Splenectomy by the laparoscopic approach. Report of a case. Presse Med. 1991;20(44):2263.

4. Targarona EM, Espert JJ, Cerdan G, Balague C, Piulachs J, Sugranes G, et al. Effect of spleen size on splenectomy outcome. A comparison of open and laparoscopic surgery. Surg Endosc. 1999;13(6):559-562. 
5. Boddy AP, Mahon D, Rhodes M. Does open surgery continue to have a role in elective splenectomy? Surg Endosc. 2006;20(7):1094-1098.

6. Maddison FE. Embolic therapy for hypersplenism. Invest Radiol. 1973;8(4):280-281.

7. Iwase K, Higaki J, Yoon HE, Mikata S, Miyazaki M, Nishitani A, et al. Splenic artery embolization using contour emboli before laparoscopic or laparoscopically assisted splenectomy. Surg Laparosc Endosc Percutan Tech. 2002;12(5):331-336.
8. Naoum JJ, Silberfein EJ, Zhou W, Sweeney JF, Albo D, Brunicardi $\mathrm{FC}$, et al. Concomitant intraoperative splenic artery embolization and laparoscopic splenectomy versus laparoscopic splenectomy: comparison of treatment outcome. Am J Surg. 2007;193(6):713-718.

9. Iwase K, Higaki J, Mikata S, Tanaka Y, Yoshikawa M, Hori S, et al. Laparoscopically assisted splenectomy following preoperative splenic artery embolization using contour emboli for myelofibrosis with massive splenomegaly. Surg Laparosc Endosc Percutan Tech. 1999;9(3):197-202. 